\title{
On the number of singular points of factorial terminal Fano threefolds
}

\author{
Yu. Prokhorov
}

UDK: 512.7

Keywords: Fano variety, terminal singularity

\section{Introduction}

Throughout this paper by $X$ we denote a Fano threefold with terminal $\mathbb{Q}$-factorial singularities and $\operatorname{rk} \operatorname{Pic}(X)=1$. Let $\iota=\iota(X)$ be its Fano index and $g:=\left(-K_{X}\right)^{3} / 2+1$ be its genus. When $\iota(X) \geq 2$, more convenient invariant of a Fano threefold $X$ is its degree $d:=\left(-K_{X}\right)^{3} / \iota^{3}$. Recall that any $X$ as above has a one-parameter smoothing $\mathfrak{X}[\mathbf{7}$. Let $\mathrm{h}^{1,2}\left(\mathfrak{X}_{s}\right)$ be the Hodge number of a general fiber of $\mathfrak{X}$. Denote by $s(V)$ be the number of singular points of $V$.

The upper bounds of $s(X)$ are interesting for classification problems, in particular, for the classification of finite subgroups of the space Cremona group (see., e.g., [8], [10, [11, [14). Y. Namikawa in [7] proved the inequality

$$
s(X) \leq 20-\operatorname{rk} \operatorname{Pic}(X)+\mathrm{h}^{1,2}\left(\mathfrak{X}_{s}\right),
$$

which holds for any Fano threefold $X$ with terminal Gorenstein singularities. However, this estimate is quite rough. For Fano threefolds with non-degenerate singularities it is known the inequality $s(X) \leq \mathrm{h}^{1,2}\left(\mathfrak{X}_{s}\right)$ (see, e.g., [12, $\left.\S 10\right]$ ).

Theorem 1 ((cf. 9, Thm. 4.1])). Suppose either 1) $\iota=1$ and $g \geq 7$ or 2) $\iota=2$ and $d \geq 3$. Then $s(X) \leq \mathrm{h}^{1,2}\left(\mathfrak{X}_{s}\right)$ and this bound is attained for some $X$ having only ordinary double points.

Recall (see [4], see also the references in [13]) that $\mathrm{h}^{1,2}\left(\mathfrak{X}_{s}\right)$ takes the following values:

\begin{tabular}{|c|c|c|c|c|c|c|c|c|c|c|c|c|c|c|c|c|}
\hline$\iota=1 \quad g$ & 12 & 10 & 9 & 8 & 7 & 6 & 5 & 4 & 3 & 2 & $\iota=2 \quad d$ & 5 & 4 & 3 & 2 & 1 \\
\hline $\mathrm{h}^{1,2}$ & 0 & 2 & 3 & 5 & 7 & 10 & 14 & 20 & 30 & 52 & $\mathrm{~h}^{1,2}$ & 0 & 2 & 5 & 10 & 21 \\
\hline
\end{tabular}

Note that in our theorem we do not assume that the singularities of $X$ are nondegenerate. Moreover, our construction allows to get better bounds in degenerate cases. If $\iota=1, g=6$ or $\iota=d=2$, then under the additional assumption that the variety $X$ has a $c A_{1}$-point, our computations allow us to get the estimate $s(X) \leq 15$ (cf. [9, proof of 1.3]). However, we do not claim that this bound is sharp.

This work is supported by the Russian Science Foundation under grant 14-50-00005. 
A Sarkisov link is the following diagram

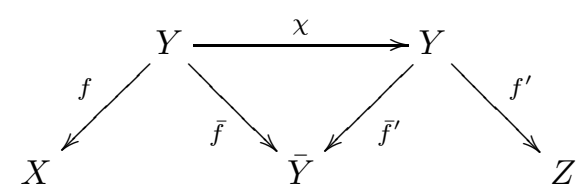

where $f$ and $f^{\prime}$ are extremal Mori contractions and $\chi$ is a flop. In our situation, the morphism $f$ is birational. Let $E$ be the exceptional divisor and $E^{\prime} \subset Y^{\prime}$ be its proper transform. If $f^{\prime}$ is also birational, then we denote by $D^{\prime}$ its exceptional divisor and $\Gamma:=$ $f^{\prime}\left(D^{\prime}\right)$ If, moreover, $\Gamma$ is curve, then it is irreducible, contained in the non-singular locus of $Z, f^{\prime}$ is the blowup of $\Gamma$ and the singularities $\Gamma$ are planar (if there are any, see [2]).

\section{Birational transformations in singular points.}

Recall that a three-dimensional singularity $P \in X$ is of type $c A_{1}^{m}$, if it is analytically equivalent to a hypersurface singularity $x_{1} x_{2}+x_{3}^{2}+x_{4}^{m}$. The blowup $f: Y \rightarrow X$ of the the maximal ideal $\mathfrak{m}_{P, X}$ of such a point is a Mori contraction (see [2]). If the $m \leq 2$, then $Y$ is smooth along $f^{-1}(P)$. If $m>2$, then $Y$ has on $f^{-1}(P)$ exactly one singular point and this point is of type $c A_{1}^{m-2}$.

Sarkisov links with centers at singular points $P \in X$ of type $c A_{1}$ appeared earlier in different papers (see, e.g., [3], [5], [1, §3], [15]) but usually under some additional restrictions. My systematize and generalize this information:

Theorem 2. Suppose that $X$ has a cA-point $P \in X$. Let $f: Y \rightarrow X$ be the blowup of $\mathfrak{m}_{P, X}$. If $\iota=2, d \geq 2$, then $f$ is included in the Sarkisov link (1), where $\chi$ is an isomorphism and $f^{\prime}$ is defined by the linear system $\left|\frac{1}{2}\left(-K_{Y^{\prime}}-E^{\prime}\right)\right|$. Except for the case $3^{\circ}$, the morphism $f^{\prime}$ contracts a divisor $D^{\prime}$ to a curve $\Gamma$ :

\begin{tabular}{|c|c|l|c|c|}
\hline$N$ & $d$ & $Z$ & $\operatorname{deg} \Gamma$ & $p_{a}(\Gamma)$ \\
\hline $1^{\circ}$ & 4 & a smooth quadric in $\mathbb{P}^{4}$ & 4 & 1 \\
$2^{\circ}$ & 3 & $\mathbb{P}^{3}$ & 6 & 4 \\
\hline $3^{\circ}$ & 2 & $\mathbb{P}^{2}, \quad f^{\prime}$ is a conic bundle with discriminant curve $\Delta \subset \mathbb{P}^{2}, \operatorname{deg} \Delta=6$ \\
\hline
\end{tabular}

If $\iota=1, g \geq 4$, then $f$ is included in the Sarkisov link (1), where, except for the case $10^{\circ}$, the morphism $f^{\prime}$ is defined by the linear system $\left|-K_{Y^{\prime}}-E^{\prime}\right|$, and, except the cases $8^{\circ}$ and [9. $f^{\prime}$ contracts a divisor $D^{\prime}$ to a curve $\Gamma$ :

\begin{tabular}{|c|c|l|c|c|}
\hline № & $g$ & \multicolumn{1}{|c|}{$Z$} & $\operatorname{deg} \Gamma$ & $p_{a}(\Gamma)$ \\
\hline $4^{\circ}$ & 10 & a non-singular Fano threefold with $\iota=2, d=5$ & 6 & 1 \\
$5^{\circ}$ & 9 & a $\mathbb{Q}$-factorial Fano threefold with $\iota=2, d=4$ & 4 & 0 \\
$6^{\circ}$ & 8 & a smooth quadric in $\mathbb{P}^{4}$ & 8 & 4 \\
$7^{\circ}$ & 7 & $\mathbb{P}^{3}$ & 8 & 6 \\
\hline $8^{\circ}$ & 6 & $\mathbb{P}^{2}, \quad f^{\prime}$ is a conic bundle with discriminant curve $\Delta \subset \mathbb{P}^{2}, \operatorname{deg} \Delta=6$ \\
$9^{\circ}$ & 5 & $\mathbb{P}^{1}, \quad f^{\prime}$ is a del Pezzo fibration of degree 4 & 4 & 0 \\
\hline $10^{\circ}$ & 4 & a $\mathbb{Q}$-factorial Fano threefold with $\iota=1, g=4$ \\
\hline
\end{tabular}

In the case $10^{\circ}, D^{\prime} \in\left|-K_{Y^{\prime}}-E^{\prime}\right|$.

Proof. In all our cases, the linear system $\left|-K_{X}\right|$ is very ample and defines an embedding $X \subset \mathbb{P}^{g+1}$ as a variety of degree $2 g-2$. Consider the projection $\psi: X \rightarrow Y_{0} \subset \mathbb{P}^{g}$ from $P$. The blowup $f: Y \rightarrow X$ of the maximal ideal $\mathfrak{m}_{P, X}$ resolves the indeterminacy points of $\psi$ and gives a morphism $Y \rightarrow Y_{0}$. Note that the exceptional divisor $E \subset Y$ is isomorphic to an irreducible quadric $Q \subset \mathbb{P}^{3}$ and $\mathscr{O}_{E}(E) \simeq \mathscr{O}_{Q}(-1)$ (see [2]). It is easy 
to see that $K_{Y}=f^{*} K_{X}+E$. This shows that the morphism $Y \rightarrow Y_{0}$ is given by the anticanonical linear system and $-K_{Y}^{3}=-K_{X}^{3}-2>0$. Therefore, $\operatorname{dim} Y_{0}=3$ and in the Stein factorization $Y \rightarrow \bar{Y} \rightarrow Y_{0}$, the morphism $\bar{f}: Y \rightarrow \bar{Y}$ is birational and crepant. Since there are at most a finite number of lines passing through $P$, the exceptional locus of the projection $\psi$ is at most one-dimensional. Thus, $\bar{f}: Y \rightarrow \bar{Y}$ is a small crepant contraction. Hence there exists a flop $\chi: Y \rightarrow Y^{\prime}$ and an extremal Mori contractions $f^{\prime}: Y^{\prime} \rightarrow Z$. Using the restriction exact sequences to $E \simeq Q \subset \mathbb{P}^{3}$, it is easy to compute that $\operatorname{dim}\left|-K_{Y^{\prime}}-E^{\prime}\right| \geq \operatorname{dim}\left|-K_{X}\right|=g-4$ always and $\left|\frac{1}{2}\left(-K_{Y^{\prime}}-E^{\prime}\right)\right| \geq d$ for $\iota=2$. Using this and solving the corresponding Diophantine equations, as in [4, ch. 4] and [9, §4], we obtain the possibilities $1^{\circ}, 10^{\circ}$. The smoothness of $Z$ in the cases $1^{\circ} 4^{\circ}$ and $6^{\circ}$ follows from $\mathbb{Q}$-factoriality (see Corollary 2 below). If $\iota=2$, then the threefold $X$ does not contain lines (for the anticanonical embedding). Therefore, the divisor $-K_{Y}$ is ample and $Y \simeq Y^{\prime}$. This proves Theorem 2 ,

REMARK 1. Let the pair $(Z, \Gamma)$ be such as in the cases $1^{\circ} 2^{\circ}, 4^{\circ} 7^{\circ}$ Suppose that

(i) the curve $\Gamma$ has planar singularities and is contained in the non-singular locus of $Z$

(ii) $\Gamma$ is a scheme-theoretic intersection of elements of the linear system $|\iota(Z) L|$, where $L$ is the ample generator of $\operatorname{Pic}(Z) \simeq \mathbb{Z}$,

(iii) the number of $\iota(Z)$-secant lines of $\Gamma$ is at most finite.

Then the blowup of $\Gamma$ can be completed to a Sarkisov link with the corresponding Fano threefold $X$ (i.e. the construction (11) can be reversed). Indeed, it follows from the condition (i) that $Y^{\prime}$ has only terminal factorial singularities, (ii) that $\left|-K_{Y^{\prime}}\right|$ has no base points, and (iii) guarantees that the corresponding morphism defines a crepant small contraction. The rest is similar to the proof of Theorem 2

Corollary 1. Suppose that in the conditions of Theorem 1, the threefold $X$ has a point of type $c A_{1}$. Then $s(X) \leq \mathrm{h}^{1,2}\left(\mathfrak{X}_{s}\right)$ and this bound is attained for some $X$ having only ordinary double points.

Proof. Note that three-dimensional terminal flops preserve types of singularities. Therefore, in the notation of Theorem 2 and (1) we have

$$
s(X) \leq s(Y)+1=s\left(Y^{\prime}\right)+1=s(Z)+s(\Gamma)+1 \leq s(Z)+p_{a}(\Gamma)+1
$$

(see [9, 5.1(ii)]). In all cases $1^{\circ} \mid 7^{\circ}$ we can choose $Z$ and $\Gamma$ so that equalities are attained. This proves Corollary 1

\section{Proof of Theorem 1; the case $g \neq 7$}

For $\iota=1, g \geq 9$, Theorem 1 was proved in [9, 1.3], and for $\iota=1, g=8$ and $\iota=2$, $d=3$ the assertion follows directly from Corollary 1 and the following lemma.

Lemma 1. Assume either: 1) $\iota=1$ and $g=8$, or 2) $\iota=2$ and $d=3$. If $s(X)>2$, then $X$ has a singularity of type $c A_{1}$.

Proof. In the case $g=8$, according to [9, 4.1], the double projection from a line contained in the non-singular locus determines a Sarkisov link, where $f^{\prime}$ is a conic bundle over $\mathbb{P}^{2}$ with discriminant curve $\Delta$ of degree 5. Similarly, for a cubic hypersurface with $\mathbb{Q}$-factorial terminal singularities the usual projection from a line defines a Sarkisov link, where $f^{\prime}$ is conic bundle over $\mathbb{P}^{2}$ with discriminant curve $\Delta$ degree 5 (in this case $\chi$ is isomorphism). Moreover, $Y^{\prime}$ has the same collection of singularities as $X$. Assume that $s(X)>2$ and all the singularities of $X$ are worse than $c A_{1}$. Let $R \in Y^{\prime}$ be a singular point and $o:=f^{\prime}(R)$. 
We claim that $R$ is the only singular point of $Y^{\prime}$ lying on the fiber $f:=f^{-1}(o)$ and the singularity of the curve $\Delta$ at $o$ is of multiplicity $\geq 3$. Let $U \subset Z=\mathbb{P}^{2}$ be a small analytic neighborhood of $o$ and $Y_{U}^{\prime}:=f^{\prime-1}(U)$. Then $Y_{U}^{\prime}$ can be embedded to $\mathbb{P}_{x_{0}, x_{1}, x_{2}}^{2} \times U_{u_{1}, u_{2}}$ and defined there by a homogeneous equation of degree 2 with respect to the variables $x_{0}, x_{1}, x_{2}$. We may assume that $R$ has coordinates $(0: 0: 1 ; 0,0)$.

If $F$ is a reducible conic, then the equation of $Y_{U}^{\prime}$ can be written in the form $x_{0} x_{1}+$ $\alpha\left(u_{1}, u_{2}\right) x_{2}^{2}=0$, where $\alpha \in \mathfrak{m}_{o, U}^{3}$. In this case $R$ is the only singular point of $Y_{U}^{\prime}$ and the discriminant curve $\Delta=\{\alpha=0\}$ has at $o$ a singularity of multiplicity $\geq 3$.

If $F$ is a double line, then the equation of $Y_{U}^{\prime}$ can be written in the form $x_{0}^{2}+\alpha x_{1}^{2}+$ $\beta x_{1} x_{2}+\gamma x_{2}^{2}=0$, where $\alpha \in \mathfrak{m}_{o, U}, \beta, \gamma \in \mathfrak{m}_{o, U}^{2}$. Since singularities of $Y_{U}^{\prime}$ are isolated, we have $\alpha \notin \mathfrak{m}_{o, U}^{2}$. This means that $R$ is the only singular point of $Y_{U}^{\prime}$. The discriminant curve $\Delta$ is given by $\beta^{2}=4 \alpha \gamma$ and again has at $o$ a singularity of multiplicity $\geq 3$. This proves our assertion.

Thus, $s\left(Y^{\prime}\right)$ is less or equal to the number of points of multiplicity $\geq 3$ of the curve $\Delta$. On the other hand, a plane (possibly reducible) curve of degree 5 has at most two triple points. Lemma 1 is proved.

It remains to consider the case $\iota=2, d \geq 4$. The following lemma is well known in the non-singular case (see, e.g., [4]). A singular case is similar.

Lemma 2. Let $X=X_{d} \subset \mathbb{P}^{d+1}$ be a Fano threefold with $\iota=2, d \geq 4$ and let $l$ be a line contained in the non-singular locus of $X$. Then the projection from l determines a Sarkisov link, where $\chi$ is an isomorphism. Moreover,

(i) if $d=4$, then $Z \simeq \mathbb{P}^{3}$, and $f^{\prime}$ is the blowup of an (irreducible) curve of degree 5 and arithmetic genus 2 lying on a quadric;

(ii) if $d=5$, then $Z$ is smooth quadric in $\mathbb{P}^{4}$ and $f^{\prime}$ is the blowup of a smooth rational cubic curve lying on a hyperplane section.

Corollary 2. If $\iota=2, d=5$, then $X$ is smooth. Let $\iota=2, d=4$. Then $s(X) \leq 2$ and if $s(X)=2$, then both singularities of $X$ are of type $c A_{1}$.

\section{The case $g=7$}

Theorem 3. Let $\iota=1, g=7$ and let $C \subset X$ be a sufficiently general conic. Then there exists a Sarkisov link (1D) with center $C$ and there are two possibilities:

(i) $Z \subset \mathbb{P}^{4}$ is a smooth quadric, $f^{\prime}$ is the blowup of a curve $\Gamma \subset Z$ with $\operatorname{deg} \Gamma=10$, $p_{a}(\Gamma)=7$.

(ii) $Z \subset \mathbb{P}^{4}$ is a cubic and $f^{\prime}$ is the blowup of a smooth rational curve $\Gamma \subset Z$ of degree 4 .

The proof substantially is similar to [4, §4.4] but in the singular case some of the arguments must be modified. According to [7] and [4, 4.2.5] the family $\mathscr{C}$ parametrizing non-degenerate conics on $X$ is not empty and two-dimensional, and according to [6. A.1.2] conics from the family $\mathscr{C}$ cover an open subset in $X$. Further, you need the following.

Lemma 3. (i) For each line $l \subset X$ there are only a finite number of lines on $X$ meeting $l$.

(ii) Let $l$ be a line contained in the non-singular locus of $X$. Then it meets a onedimensional family of conics.

(iii) There is a non-degenerate conic contained in the non-singular locus of $X$.

Proof. (i) follows from the fact that $\operatorname{dim}|H-2 l| \geq 1$ and $\operatorname{Pic}(X)=\mathbb{Z} \cdot[H]$. 
(ii) Consider the double projection from $l$ and the corresponding Sarkisov link [9]. In the notation of (1), the morphism $f$ is the blowup of $l, Z \simeq \mathbb{P}^{1}$ and $f^{\prime}$ is a del Pezzo fibration of degree 5. The proper transform $C^{\prime} \subset Y^{\prime}$ of a conic $C \subset X$ meeting $l$ is a line in a fiber of $f^{\prime}$ (because $-K_{Y^{\prime}} \cdot C^{\prime}=1$ and $E^{\prime} \cdot C^{\prime} \geq 1$ ). There is at most a one-dimensional family of such lines. This proves (ii).

(iii) Assume that all of the conics from the (two-dimensional) family $\mathscr{C}$ pass through a point $P \in X$. Take line $l \subset X$ that does not pass through $P$ and all the lines $l_{i}$ meeting $l$ also do not pass through $P$. Then the map $\chi \circ f^{-1}: X \rightarrow Y^{\prime}$ is an isomorphism near $P$. The line $l$ intersects a one-dimensional family of conics from the family $\mathscr{C}$ and proper transforms these conics on $Y^{\prime}$ are lines in the fiber of $f^{\prime}$ passing through the point $P^{\prime}:=\chi\left(f^{-1}(P)\right)$. Hence this fiber is a cone in $\mathbb{P}^{5}$ with vertex $P^{\prime}$. But since the singularities $Y^{\prime}$ are hypersurface, the dimension of the tangent space to a fiber of $f^{\prime}$ is at most 4 . The contradiction proves our lemma.

Thus there exists a conic $C$ contained in the non-singular locus of $X$. Take such a conic so that it is not contained in the surface swept out by lines. Consider the projection of $\psi: X \rightarrow Y_{0} \subset \mathbb{P}^{5}$ from the linear span of $C$. Since $X \cap\langle C\rangle=C$, the blowup $f: Y \rightarrow X$ of the conic $C$ resolves the indeterminacy points of $\psi$ and defines a morphism $f_{0}: Y \rightarrow Y_{0}$. Here in the Stein factorization $Y \rightarrow \bar{Y} \rightarrow Y_{0}$, the morphism $\bar{f}: Y \rightarrow \bar{Y}$ is birational and is given by a multiple of the linear system $\left|-K_{Y}\right|$, i.e., $\bar{f}$ is crepant.

Lemma 4. The exceptional locus of $\bar{f}$ is one-dimensional.

Proof. Suppose that $\bar{f}$ contracts a divisor $D$. It is easy to compute (see [4, 4.1.2]) that $D \sim \alpha\left(2\left(-K_{Y}\right)-3 E\right)$ for some $\alpha \in \mathbb{Z}_{>0}$ and $D \cdot E \cdot\left(-K_{Y}\right)=14 \alpha>0$. In particular, $\Lambda:=f_{0}(D \cap E)$ is a curve. Since the conic $C$ meets only a finite number of lines, a general fiber of $D$ must be a conic meeting $C$ at two points [4, 4.4.1]. If the restriction $\left.f_{0}\right|_{E}: E \rightarrow f_{0}(E)$ is a birational morphism, $f_{0}(E)$ is a ruled surface of degree 4 singular along $\Lambda$, where

$$
\operatorname{deg} \Lambda=\frac{1}{2} D \cdot E \cdot\left(-K_{Y}\right)=7 \alpha \geq 7
$$

This is impossible, see e.g. [4, 4.4.8]. Therefore $f_{0}$ is a morphism of degree $2, f_{0}(E)$ is a quadric, and $X_{0} \subset \mathbb{P}^{5}$ is a subvariety of degree 3. Since $\operatorname{rk} \operatorname{Cl}(\bar{Y})=\operatorname{rk} \operatorname{Cl}\left(Y_{0}\right)=1$, the variety $Y_{0}$ is a cone over a rational twisted cubic curve with a vertex in a line. However, this cone contains no quadrics. The contradiction proves Lemma 4

Further, as in [4, §4.1], there exists a flop $\chi: Y \rightarrow Y^{\prime}$ and an extremal Mori contraction $f^{\prime}: Y^{\prime} \rightarrow Z$. By solving the corresponding Diophantine equations, as in [4, ch. 4] or [9. $\S 4$ ], we obtain the possibilities (i) (ii). Theorem 3 is proved. For the proof of Theorem 1 in the case $g=7$, similar to (2), we write

$$
s(X)=s(Y)=s\left(Y^{\prime}\right)=s(Z)+s(\Gamma) \leq s(Z)+p_{a}(\Gamma) .
$$

If the possibility (i) of Theorem 3 occurs, then the variety $Z$ is smooth and therefore $s(X) \leq p_{a}(\Gamma)=7$. Otherwise the curve $\Gamma$ is smooth and therefore $s(X)=s(Z)$. Then $s(Z) \leq 5$ according to the already proved case of Theorem 1 .

\section{References}

[1] I. Cheltsov, V. Przyjalkowski, and C. Shramov. Which quartic double solids are rational? arXiv preprint, 1508.07277, 2015.

[2] S. Cutkosky. Elementary contractions of Gorenstein threefolds. Math. Ann., 280(3):521-525, 1988.

[3] J. Cutrone and N. Marshburn. Towards the classification of weak Fano threefolds with $\rho=2$. Cent. Eur. J. Math., 11(9):1552-1576, 2013. 
[4] V. Iskovskikh and Yu. Prokhorov. Fano varieties. Algebraic geometry V., volume 47 of Encyclopaedia Math. Sci. Springer, Berlin, 1999.

[5] P. Jahnke, Th. Peternell, and I. Radloff. Threefolds with big and nef anticanonical bundles II. Cent. Eur. J. Math., 9(3):449-488, 2011.

[6] A. Kuznetsov, Yu. Prokhorov, and C. Shramov. Hilbert schemes of lines and conics and automorphism groups of Fano threefolds. ArXiv e-print, 1605.02010, 2016.

[7] Y. Namikawa. Smoothing Fano 3-folds. J. Algebraic Geom., 6(2):307-324, 1997.

[8] Yu. Prokhorov. Simple finite subgroups of the Cremona group of rank 3. J. Algebraic Geom., 21(3):563600, 2012.

[9] Yu. Prokhorov. On G-Fano threefolds. Izv. Ross. Akad. Nauk Ser. Mat., 79(4):159-174, 2015.

[10] Yu. Prokhorov and C. Shramov. Jordan constant for Cremona group of rank 3. ArXiv e-print, 1608.00709, 2016.

[11] Yu. Prokhorov and C. Shramov. p-subgroups in the space Cremona group. ArXiv e-print, 1610.02990, 2016.

[12] Yu. Prokhorov. G-Fano threefolds, I. Adv. Geom., 13(3):389-418, 2013.

[13] V. Przyjalkowski and C. Shramov. On Hodge numbers of complete intersections and Landau-Ginzburg models. Int. Math. Res. Not., 2015(21):11302-11332, 2015.

[14] V.Przyjalkowski and C. Shramov. Double quadrics with large automorphism groups. Proc. Steklov Inst. Math., 294:154-175, 2016.

[15] K. Takeuchi. Weak Fano threefolds with del Pezzo fibration. ArXiv e-print, 0910.2188, 2009.

Steklov Mathematical Institute of Russian Academy of Sciences (Moscow)

E-mail address: prokhoro@mi.ras.ru 